\title{
National Football League Wide Receivers and Running Backs Have Decreased Production Following ACL Reconstruction: An Evaluation of Fantasy Football Performance as an Outcome Measure
}

\author{
Colin J. Burgess, D.O., Vivek Singh, M.D., M.P.H., Katherine A. Lygrisse, M.D., \\ Kenneth Choy, B.A., Randy M. Cohn, M.D., and Adam Bitterman, D.O.
}

\begin{abstract}
Purpose: To use fantasy football points as a simple measure alongside on-field statistics to compare performance in National Football League (NFL) offensive skill position players before and after anterior cruciate ligament (ACL) reconstruction. Methods: A retrospective review of all NFL quarterbacks (QB), running backs (RB), wide receivers (WR), and tight ends (TE) who sustained an isolated, unilateral ACL injury from 1988 to 2017 was conducted. Data were collected from public data sources, team releases, NFL injury reports, press releases, and other Internet resources. For each player, a matched control with similar demographics was identified. Their in-game performance post-ACL reconstruction was analyzed using fantasy football points as an outcome measure. Results: A total of 13 QBs, 30 RBs, and 29 WRs who underwent ACL reconstruction from 1988 to 2017 and who met inclusion criteria were retrospectively identified and reviewed. Of the 13 quarterbacks included in the study, there was no statistically significant difference in fantasy football points between the pre- and post-ACL reconstruction groups, as well as post-ACL and matched control groups. There was a statistically significant decrease in career fantasy football performance of running backs post-ACL reconstruction compared with matched control groups (129.6 vs 553.6; $P<.0001)$. There was also a statistically significant decrease in per game fantasy football points post-ACL reconstruction (4.4 vs 11.2; $P<.0001)$. Lastly, WRs also demonstrated a decrease in career fantasy football performance post-ACL reconstruction compared with matched controls (145.3 vs 460.9; $P=.002)$. In addition, they also had a decrease in per game fantasy football performance $(5.0$ vs $7.7 ; P=.042)$. Conclusions: Quarterbacks did not have a statistically significant decrease in performance following ACL reconstruction based on fantasy football performance. Conversely, both running backs and wide receivers had decreased per game and career performance post-ACL reconstruction based on their fantasy football statistics. Furthermore, RBs had the largest decline in production each season over a 3-year period following ACLR compared to QBs and WRs, respectively. Level of Evidence: Level III, case-control study.
\end{abstract}

Donald and Barbara Zucker School of Medicine at Hofstra/Northwell, Plainview, New York, U.S.A. (C.J.B., K.A.L., R.M.C., A.B.); New York University Langone Health, New York, New York, U.S.A. (V.S., K.A.L); and New York Institute of Technology - College of Osteopathic Medicine, Old Westbury, New York, U.S.A. (K.C.).

The authors report no conflicts of interest in the authorship and publication of this article.

Full ICMJE author disclosure forms are available for this article online, as supplementary material.

Received May 28, 2021; accepted September 28, 2021.

Address correspondence to Colin J. Burgess, D.O., 888 Old Country Rd., Plainview, NY 11803,U.S.A. E-mail: CBurgess1@Northwell.edu

(C) 2021 THE AUTHORS. Published by Elsevier Inc. on behalf of the Arthroscopy Association of North America. This is an open access article under the CC BY-NC-ND license (http://creativecommons.org/licenses/by-nc-nd/4.0/). 2666-061X/21804

https://doi.org/10.1016/j.asmr.2021.09.021

\section{Introduction}

A nterior cruciate ligament (ACL) injury is a common and devastating injury, particularly in contact sports like American football. ${ }^{1-6}$ As a result, understanding the impact of anterior cruciate ligament reconstruction (ACLR) in American football athletes is crucial to counseling patients after this injury and improving outcomes. Results of previous studies suggest that ACLR leads to a significant decrease in level of performance and career longevity among National Football League (NFL) athletes. ${ }^{7,8}$ Among NFL players, offensive skill position players have been shown to possess the highest risk of ACL injury. ${ }^{9}$ Additionally, many parameters and outcome measures have been used for evaluating performance after ACLR in this population, some of which include games played, 
games started, in-game statistics, and multiple efficiency ratios used to condense a player's stats into individual numbers. ${ }^{2,7,10-12}$ Despite many studies investigating this topic, a universal consensus to best compare a player's performance following ACLR remains an area of debate.

Fantasy sports is a game in which participants assemble virtual teams of real players in a professional sport and compete on the basis of the statistical performance of those players in actual games. It is estimated that $\sim 60$ million people in North America participate in fantasy sports annually, making fantasy football performance a generalizable metric of on-field performance. ${ }^{13}$ Fantasy football points are a single numerical value used to assess a players' performance. Each offensive player accumulates points based on their on-field statistics. Therefore, we believe that these fantasy football points are a generalizable way to concisely evaluate performance in NFL players, including pre- and post-ACLR. This metric is likely to be more readily understood by patients compared to the traditionally used patient-reported outcome measures.

The purpose of this study is to use fantasy football points as a simple measure alongside on-field statistics to compare performance in NFL offensive skill position players pre- and post-ACLR. We hypothesize that the statistical performance based on fantasy football points of NFL offensive players will decrease after undergoing ACLR.

\section{Methods}

A retrospective review of all NFL quarterbacks (QB), running backs (RB), wide receivers (WR), and tight ends (TE), who sustained an isolated, unilateral ACL injury from 1988 to 2017 was conducted using public injury data, from team releases, NFL injury reports, press releases, and other internet resources. ${ }^{4,5,8-11}$ These data are available to the public; therefore, this study did not require approval from our institutional review board (IRB). Players who played at least three regular-season NFL games prior to their injury and at least three regular season NFL games after their injury were included in this analysis. Players with a prior ACL injury, concomitant ligamentous/meniscal injuries in the ipsilateral knee, or other reported concurrent injuries regardless of location were excluded from our analysis. Although regarded as an offensive skill position, tight ends were excluded from this study due to a small number of subjects for analysis.

Demographic data, such as age, body mass index (BMI; $\mathrm{kg} / \mathrm{m}^{2}$ ), date of injury (DOI), date of return to play (RTP), and NFL experience were collected. Performance variables pertaining to regular-season play for the three-position groups both prior and after the injury year were also collected. For QBs, the performance variables collected included games played, starts, completions, pass attempts, passing yards, touchdowns thrown, interceptions, rush attempts, rushing yards, and rush touchdowns. For RBs, the performance variables collected included games played, starts, rushing attempts, rushing yards, rushing touchdowns, receptions, receiving yards, receiving touchdowns, and time to return. For WRs, the performance variables collected included games played, starts, targets, receptions, catch percentage, receiving yards per reception, total receiving yards, touchdowns, yards per target, and time to return. These values were then converted to fantasy football points using the standard Entertainment and Sports Programming Network (ESPN) fantasy point system ${ }^{14}$ through the use of unbiased in-game statistics available on STATS.com and Pro-Football-Reference.com (Fig 1). Official NFL personnel collect all on-field statistics live, which are then reviewed and edited post-game to ensure the correct statistics are logged for each participating player. No underestimation or overestimation of fantasy points is possible, as they are solely dependent on the in-game statistics and performance of the player. ${ }^{15}$

Each QB, WR, and RB in the ACL injury cohort was matched to a player without a documented history of an ACL injury (control). Matching was based on the following parameters in decreasing order of importance: prior NFL seasons played, individual (nonaverage) performance statistics prior to index year, and BMI. ${ }^{4,5,7-10}$ Index year was defined as a certain time point for each control that was analogous to the time at which an ACL injury occurred in the injury cohort. For example, if a player suffered an ACL injury 2 years into his NFL career, the index year for the control would be

\begin{tabular}{|lc|}
\hline \multicolumn{1}{|c|}{ Ccoring } & Points \\
\hline \multicolumn{1}{|c|}{ Category } & \\
\hline Passing & 6 \\
\hline TD Pass & 1 \\
Every 25 Passing Yards & 2 \\
2-point Conversion & -2 \\
Interception Thrown & \\
\hline Rushing & 6 \\
TD Rush & 1 \\
Every 10 Rushing Yards & 2 \\
2-point Rushing Conversion & \\
\hline Receiving & 6 \\
\hline TD Reception & 1 \\
Every 10 Receiving Yards & 2 \\
2-point Receiving Conversion & \\
\hline Miscellaneous Offense & 6 \\
\hline Kickoff Return TD & 6 \\
Punt Return TD & 6 \\
Fumble Recovered for TD & -2 \\
Each Fumble lost & \\
\hline TD: Touchdown &
\end{tabular}

Fig 1. Standard fantasy football scoring. 
Table 1. QB Pre-ACL Reconstruction in Cases Versus Preindex Year in Controls

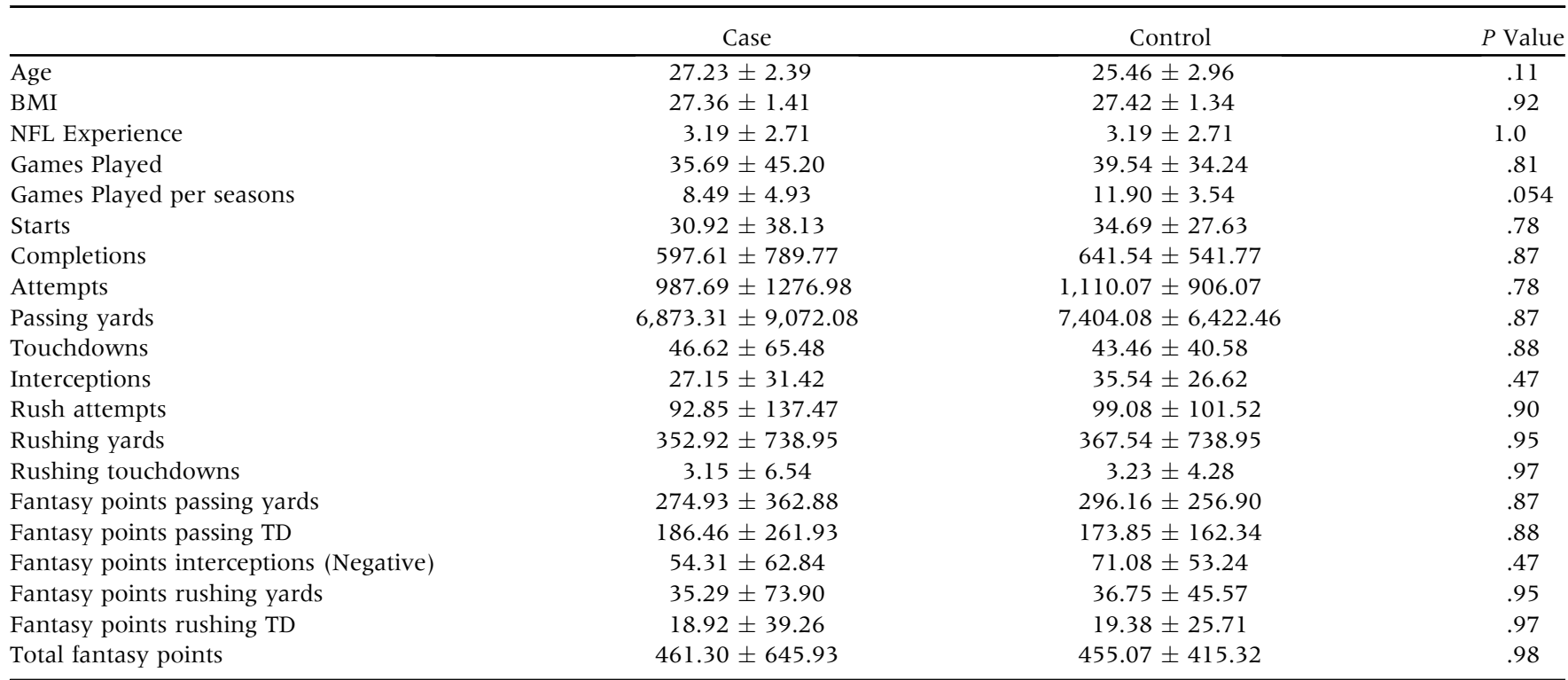

ACL, anterior cruciate ligament; BMI, body mass index; NFL, National Football League; QB, quarterback; TD, touchdowns.

two years into the control athlete's career. The same demographic and performance variables were collected for both cohorts, and mean performance over time was calculated.

Mean performance values preinjury and preindex year were compared for each position group as were the postinjury and postindex year mean performance values. Preinjury and postinjury mean performance values were compared for each position group in both cohorts. Lastly, mean performance values were compared between both cohorts for each of the first three seasons following injury or index year for each position group, respectively.

\section{Statistical Analysis}

All data were organized and collected on Excel software (Microsoft Corporation, Richmond, WA). Continuous variables were evaluated and represented as means \pm SD. Independent-sample, two-sided $t$-tests were used to test for significant differences. All statistical analyses and calculations were performed using SPSS v25 (IBM Corporation, Armonk, New York). A P value of less than .05 was used to determine the statistical significance of a finding. In addition, a post hoc power analysis was performed using $\mathrm{G}^{*}$ Power (version 3.1.9.7) to ensure adequate statistical power ( $\geq 80 \%$ ) was achieved to fully elucidate significant findings.

Table 2. QB Pre-ACL Reconstruction Versus Post-ACL Reconstruction in Cases

\begin{tabular}{lccc}
\hline & Before & After & $P$ Value \\
\hline NFL experience & $3.19 \pm 2.71$ & $6.23 \pm 3.27$ & .016 \\
Games played & $35.69 \pm 45.20$ & $58.00 \pm 47.68$ \\
Games played per seasons & $8.49 \pm 4.93$ & $8.30 \pm 3.74$ & .23 \\
Starts & $30.92 \pm 38.13$ & $42.69 \pm 54.41$ & .53 \\
Completions & $597.62 \pm 789.77$ & $940.38 \pm 1,223.36$ \\
Attempts & $987.69 \pm 1,276.98$ & $1,526.08 \pm 1,922.55$ \\
Passing yards & $6,873.31 \pm 9,072.08$ & $11,191.46 \pm 14,671.23$ \\
Touchdowns & $46.62 \pm 65.48$ & $68.08 \pm 99.72$ \\
Interceptions & $27.15 \pm 31.42$ & $40.46 \pm 45.10$ \\
Rush attempts & $92.85 \pm 137.47$ & $87.00 \pm 101.46$ \\
Rushing yards & $352.92 \pm 738.95$ & $205.15 \pm 289.19$ \\
Rushing touchdowns & $3.15 \pm 6.54$ & $2.54 \pm 4.14$ \\
Fantasy points passing Yards & $274.93 \pm 362.88$ & $447.66 \pm 586.85$ \\
Fantasy points passing TD & $186.46 \pm 261.93$ & $272.31 \pm 398.86$ \\
Fantasy points interceptions (Negative) & $54.31 \pm 62.84$ & $80.92 \pm 90.21$ \\
Fantasy points rushing yards & $35.29 \pm 73.90$ & .52 \\
Fantasy points rushing TD & $18.92 \pm 39.26$ & .39 \\
Total fantasy points & $461.30 \pm 645.93$ & .90 \\
\hline
\end{tabular}

Bold value is statistically significant $P<.05$.

NFL, National Football League; TD, touchdowns. 
Table 3. QB Post-ACL reconstruction in Cases Versus Postindex Year in Controls

\begin{tabular}{|c|c|c|c|}
\hline & After-ACL & Post-Index Year & $P$ Value \\
\hline$\overline{\text { NFL Experience }}$ & $6.23 \pm 3.27$ & $8.54 \pm 5.15$ & .19 \\
\hline Games Played & $58.00 \pm 47.68$ & $95.69 \pm 77.96$ & .15 \\
\hline Games Played per seasons & $8.30 \pm 3.74$ & $9.29 \pm 4.54$ & .55 \\
\hline Completions & $940.38 \pm 1,223.36$ & $1,718.77 \pm 1,623.89$ & .18 \\
\hline Attempts & $1,526.08 \pm 1,922.55$ & $2,910.77 \pm 2,700.94$ & .15 \\
\hline Passing Yards & $11,191.46 \pm 14,671.23$ & $20,002.54 \pm 19,129.84$ & .20 \\
\hline Rush Attempts & $87.00 \pm 101.46$ & $166.15 \pm 289.19$ & .085 \\
\hline Rushing Yards & $205.15 \pm 289.19$ & $476.85 \pm 413.40$ & .064 \\
\hline Rushing Touchdowns & $2.54 \pm 4.14$ & $4.62 \pm 4.00$ & .20 \\
\hline Fantasy Points Passing Yards & $447.66 \pm 586.85$ & $800.10 \pm 765.19$ & .20 \\
\hline Fantasy Points Passing TD & $272.31 \pm 398.86$ & $471.69 \pm 484.28$ & .26 \\
\hline Fantasy Points Interceptions (Negative) & $80.92 \pm 90.21$ & $184.31 \pm 175.24$ & .071 \\
\hline
\end{tabular}

\section{Results}

A total of 13 QBs, 30 RBs, and 29 WRs were identified as having an isolated, unilateral ACL tear between 1988 and 2017, having previously played at least three regular-season NFL games prior to their injury and went on to play at least three regularseason NFL games following their injury. A post hoc power analysis confirmed that all findings were sufficiently powered to show actual statistical differences between the cohorts.

\section{Quarterbacks}

QBs within the injury cohort had similar demographics when compared to controls. There was no significant difference in QB performance between the injury cohort and controls prior to ACL injury or index year (Table 1). Following ACLR, QBs showed no statistically significant change in fantasy points or in performance variables except for NFL experience $(P=.016)$ (Table 2). While QBs showed decreased on-field performance and fantasy football points in the index year post-ACLR, there were

Table 4. QB Cases Versus Controls: Year One to Year Three

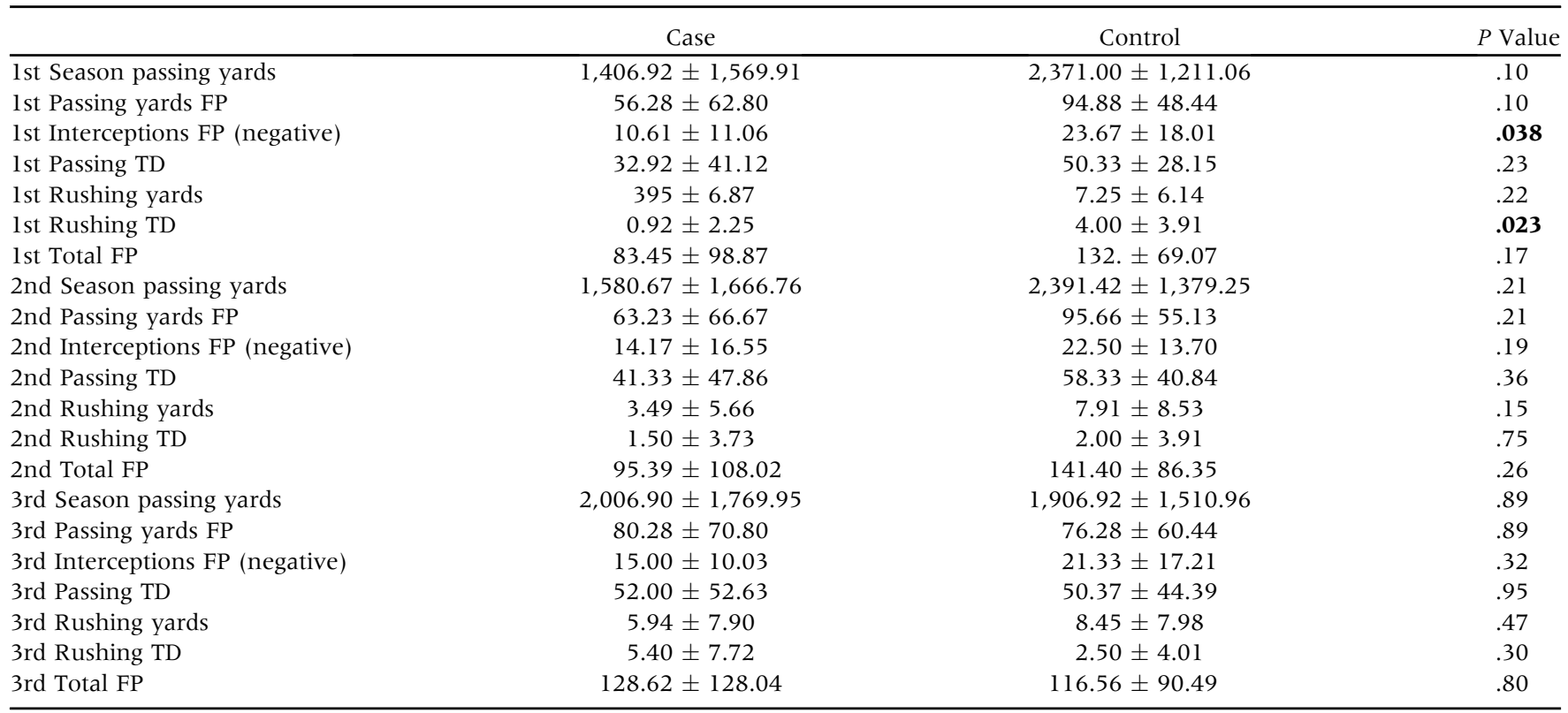

Bold values are statistically significant $P<.05$.

$\mathrm{FP}$, fantasy points; $\mathrm{QB}$, quarterback; TD, touchdowns. 
Table 5. RB Pre-ACL Reconstruction in Cases Versus Preindex Year in Controls

\begin{tabular}{|c|c|c|c|}
\hline & Case & Control & $P$ Value \\
\hline$\overline{\text { Age }}$ & $22.47 \pm 0.86$ & $22.23 \pm 1.01$ & .34 \\
\hline BMI & $30.09 \pm 1.48$ & $30.74 \pm 2.00$ & .16 \\
\hline NFL experience & $4.03 \pm 2.61$ & $4.03 \pm 2.61$ & 1.00 \\
\hline Games played per seasons & $12.77 \pm 3.24$ & $13.70 \pm 2.06$ & .19 \\
\hline Rush attempts & $461.73 \pm 413.42$ & $837.10 \pm 711.19$ & .015 \\
\hline Rush attempts per game & $8.49 \pm 5.96$ & $14.34 \pm 5.01$ & $<.0001$ \\
\hline Rushing yards per game & $36.48 \pm 26.95$ & $61.51 \pm 21.01$ & $<.0001$ \\
\hline Rushing yards per season & $487.05 \pm 370.53$ & $845.73 \pm 324.48$ & $<.0001$ \\
\hline Rushing touchdowns & $14.13 \pm 15.36$ & $26.17 \pm 23.55$ & .023 \\
\hline Receptions & $109.10 \pm 128.34$ & $123.63 \pm 105.99$ & .63 \\
\hline Receiving yards & $938.60 \pm 1120.13$ & $967.13 \pm 787.80$ & .91 \\
\hline Receiving touchdowns & $4.17 \pm 7.14$ & $3.17 \pm 3.39$ & .49 \\
\hline Fantasy points receiving touchdowns & $25.00 \pm 42.87$ & $19.00 \pm 20.37$ & .49 \\
\hline Fantasy points total points & $515.33 \pm 500.53$ & $763.37 \pm 638.08$ & .099 \\
\hline Points per game & $8.53 \pm 5.33$ & $13.31 \pm 4.69$ & .001 \\
\hline Points per season & $113.50 \pm 75.83$ & $182.28 \pm 72.18$ & .001 \\
\hline
\end{tabular}

Bold values are statistically significant $P<.05$.

ACL, anterior cruciate ligament; BMI, body mass index; NFL, National Football League; RB, running back.

no significant differences in any performance variables between the QBs in the injury cohort following ACLR (Table 3). No performance variable was statistically different between the two groups in the 2 nd and 3rd seasons, respectively (Table 4).

\section{Running Backs}

RBs in the injury and control cohorts had similar demographic information. Differences in fantasy football points and on-field statistics between the two groups are listed in Table 5. Following ACLR, RBs had a

Table 6. RB Pre-ACL Reconstruction Versus Post-ACL Reconstruction in Cases

\begin{tabular}{lccc}
\hline & Before & After & $P$ Value \\
\hline NFL experience & $4.03 \pm 2.61$ & $1.77 \pm 2.06$ & $<.0001$ \\
Games played & $53.17 \pm 39.19$ & $18.03 \pm 27.12$ & $<.01 \pm 5.57$ \\
Games played per seasons & $12.77 \pm 3.24$ & $8.33 \pm 17.68$ & $\mathbf{0 0 0 1}$ \\
Starts & $21.50 \pm 21.91$ & $\mathbf{. 0 1 3}$ \\
Rush attempts & $461.73 \pm 413.42$ & $\mathbf{. 0 0 1}$ \\
Rush attempts per game & $8.49 \pm 5.96$ & $\mathbf{. 0 0 2}$ \\
Rushing yards & $2,025.73 \pm 1,959.44$ & $4.08 \pm 4.79$ & $\mathbf{. 0 0 1}$ \\
Rushing yards per attempt & $4.24 \pm 0.59$ & $553.00 \pm 1,291.30$ \\
Rushing yards per game & $36.48 \pm 26.95$ & $2.44 \pm 1.84$ \\
Rushing touchdowns & $14.13 \pm 15.36$ & $16.19 \pm 20.71$ \\
Receptions & $109.10 \pm 128.34$ & $170.54 \pm 240.65$ \\
Receiving yards & $938.60 \pm 1,120.13$ & $26.43 \pm 38.86$ \\
Receiving touchdowns & $4.17 \pm 7.14$ & $191.67 \pm 281.13$ \\
Fantasy points rushing yards & $202.57 \pm 195.94$ & $0.83 \pm 1.29$ \\
Fantasy points touchdowns & $84.80 \pm 92.17$ & $55.30 \pm 129.13$ \\
Fantasy points receptions & $109.10 \pm 128.34$ & $23.80 \pm 52.25$ \\
Fantasy points receiving yards & $93.71 \pm 78.78$ & $26.43 \pm 38.86$ \\
Fantasy points receiving touchdowns & $25.00 \pm 42.87$ & $\mathbf{. 0 0 1}$ \\
Fantasy points total points & $515.33 \pm 500.53$ & $\mathbf{. 0 0 1}$ \\
Points per game & $8.53 \pm 5.33$ & $\mathbf{. 0 0 1}$ \\
Points per season & $113.50 \pm 75.83$ & $\mathbf{. 0 1 5}$ \\
\hline
\end{tabular}

Bold values are statistically significant $P<.05$.

ACL, anterior cruciate ligament; NFL, National Football League; RB, running back. 
Table 7. RB Post-ACL Reconstruction in Cases Versus Postindex Year in Controls

\begin{tabular}{|c|c|c|c|}
\hline & After ACL & Postindex Year & $P$ Value \\
\hline NFL experience & $1.77 \pm 2.06$ & $3.50 \pm 1.88$ & .001 \\
\hline Games played & $1.77 \pm 2.06$ & $44.70 \pm 26.42$ & $<.0001$ \\
\hline Games played per seasons & $6.51 \pm 5.57$ & $12.37 \pm 3.00$ & $<.0001$ \\
\hline Rush attempts & $132.37 \pm 286.55$ & $615.93 \pm 447.44$ & $<.0001$ \\
\hline Rush attempts per game & $4.08 \pm 4.79$ & $12.97 \pm 3.93$ & $<.0001$ \\
\hline Rushing yards & $553.00 \pm 1,291.30$ & $2,500.43 \pm 1,932.18$ & $<.0001$ \\
\hline Rushing yards per attempt & $2.44 \pm 1.84$ & $3.88 \pm 0.46$ & $<.0001$ \\
\hline Rushing yards per game & $16.19 \pm 20.71$ & $51.15 \pm 18.86$ & $<.0001$ \\
\hline Rushing touchdowns & $170.54 \pm 240.65$ & $17.77 \pm 16.10$ & .001 \\
\hline Receptions & $26.43 \pm 38.86$ & $101.43 \pm 84.89$ & $<.0001$ \\
\hline Receiving yards & $191.67 \pm 281.13$ & $789.47 \pm 787.22$ & $<.0001$ \\
\hline Receiving touchdowns & $0.83 \pm 1.29$ & $2.77 \pm 3.49$ & .006 \\
\hline Fantasy points rushing yards & $55.30 \pm 129.13$ & $250.04 \pm 193.22$ & $<.0001$ \\
\hline Fantasy points touchdowns & $23.80 \pm 52.25$ & $106.60 \pm 96.63$ & $<.0001$ \\
\hline Fantasy points receptions & $26.43 \pm 38.86$ & $101.43 \pm 94.89$ & $<.0001$ \\
\hline Fantasy points receiving yards & $19.16 \pm 28.11$ & $78.95 \pm 7872$ & $<.0001$ \\
\hline Fantasy points receiving touchdowns & $5.00 \pm 7.73$ & $16.60 \pm 20.94$ & .006 \\
\hline Fantasy points total points & $129.60 \pm 242.89$ & $553.62 \pm 446.20$ & $<.0001$ \\
\hline Points per game & $4.43 \pm 4.73$ & $11.18 \pm 4.89$ & $<.0001$ \\
\hline Points per season & $45.70 \pm 53.29$ & $142.25 \pm 74.34$ & $<.0001$ \\
\hline
\end{tabular}

Bold values are statistically significant $P<.05$.

ACL, anterior cruciate ligament; NFL, National Football League; RB, running back.

significant decrease in fantasy points and all performance variables (Table 6).

RBs had significantly worse performance and fewer fantasy points after ACLR compared to controls postindex year (Table 7). In the lst seasons after ACLR, RBs had significantly decreased rushing yards $(P<$ $.001)$, receptions $(P=.013)$, receiving yards $(P=.009)$, touchdowns $(P<.001)$ and total fantasy points $(P<$ $.001)$ compared to controls. The 2 nd season after ACLR, RBs did not statistically differ compared to controls. However, during the 3rd season after ACLR, RBs had significantly decreased rushing yards $(P=.006)$, touchdowns $(P=.018)$, and total fantasy points $(P=.032)$ compared with controls (Table 8$)$.

Table 8. RB Cases Versus Controls: Year One to Year Three

\begin{tabular}{lccc}
\hline & Case & Control & $P$ Value \\
\hline 1st season rushing & $28.37 \pm 46.70$ & $80.04 \pm 41.11$ & $<.0001$ \\
lst reception & $15.00 \pm 14.23$ & $28.67 \pm 2150$ & $\mathbf{. 0 1 3}$ \\
1st receiving & $11.29 \pm 10.82$ & $22.77 \pm 17.32$ & $\mathbf{. 0 0 9}$ \\
1st TD fantasy points & $14.73 \pm 20.93$ & $42.40 \pm 29.22$ & $<.0001$ \\
1st total fantasy points & $69.38 \pm 84.28$ & $173.87 \pm 92.42$ & $<.0001$ \\
2nd season rushing & $45.00 \pm 40.32$ & $72.50 \pm 41.42$ & .70 \\
2nd reception & $19.00 \pm 11.43$ & $32.93 \pm 23.26$ & .068 \\
2nd receiving & $13.75 \pm 7.70$ & $26.19 \pm 20.21$ & .056 \\
2nd TD fantasy points & $29.72 \pm 24.17$ & $34.22 \pm 27.94$ & .57 \\
2nd Total FP & $106.47 \pm 76.29$ & $159.91 \pm 104.88$ & .091 \\
3rd rushing & $22.83 \pm 28.20$ & $72.16 \pm 40.24$ & $\mathbf{. 0 0 6}$ \\
3rd reception & $15.86 \pm 22.15$ & $27.60 \pm 20.62$ & .21 \\
3rd receiving & $10.86 \pm 15.43$ & $21.29 \pm 16.30$ & .15 \\
3rd TD fantasy points & $8.57 \pm 10.88$ & $36.60 \pm 28.27$ & $\mathbf{. 0 1 8}$ \\
3rd Total FP & $58.11 \pm 71.87$ & $150.14 \pm 98.78$ & $\mathbf{. 0 3 2}$ \\
\hline \multicolumn{1}{c}{ Bold } & &
\end{tabular}

Bold values are statistically significant $P<.05$.

$\mathrm{FP}$, fantasy points; $\mathrm{RB}$, running back; TD, touchdowns.

\section{Wide Receivers}

WRs preinjury did not statistically differ in age, BMI, NFL experience, all performance variables, and fantasy points compared with preindex year controls (Table 9). Following ACLR, WRs had a significant decrease in NFL experience, statistical performance, and fantasy football points (Table 10).

Following ACLR, WRs had significantly decreased performance compared to postindex year controls with regard to NFL experience $(P<.001)$, number of games played $(P<.001)$, games played per season $(P=.007)$, targets $(P<.001)$, targets per game $(P=.036)$, receptions $(P=.002)$, receiving yards $(P=.002)$, receiving yards per game $(P=.042)$, receiving yards per season $(P=.046)$, touchdowns $(P=.003)$, and fantasy points in all areas (Table 11). However, when analyzing the first three seasons post-ACLR and post-index year, there were no significant performance or fantasy point differences between WRs who underwent ACLR and the controls (Table 12).

\section{Discussion}

The results of our study show that while QBs achieved statistically equivalent on-field performance following ACLR compared to their preinjury state, as well as the postindex year control group, RBs and WRs had a significant statistical decrease in production following ACLR compared to their preinjury state, as well as the postindex year control group, respectively. This difference can be appreciated in fantasy football point decreases seen in both the RB and WR groups on a per game basis, as well as for the duration of their career (Table 13). An ACL rupture is 
Table 9. WR Pre-ACL Reconstruction in Cases Versus Preindex Year in Controls

\begin{tabular}{lccc}
\hline & Case & Control & $P$ Value \\
\hline Age & $25.90 \pm 3.11$ & $25.43 \pm 3.20$ & .58 \\
BMI & $26.71 \pm 1.55$ & $26.61 \pm 1.23$ & .10 \\
NFL experience & $3.52 \pm 3.18$ & \pm 3.18 & 1.00 \\
Games played & $45.66 \pm 46.65$ & $46.59 \pm 45.80$ & .94 \\
Games played per seasons & $11.62 \pm 4.09$ & $11.70 \pm 4.76$ \\
Targets & $254.69 \pm 354.99$ & $303.21 \pm 409.52$ & $4.82 \pm 3.02$ \\
Targets per game & $4.18 \pm 2.61$ & $180.21 \pm 243.42$ \\
Reception & $150.24 \pm 217.10$ & $2.79 \pm 1.84$ & .63 \\
Reception per game & $2.41 \pm 1.61$ & $50.37 \pm 19.13$ \\
Catch\% & $52.38 \pm 17.56$ & $2,438.76 \pm 3,333.74$ \\
Receiving & $2055.86 \pm 3049.29$ & $12.27 \pm 5.11$ \\
Yards per reception & $13.13 \pm 6.30$ & $37.93 \pm 25.66$ \\
Rec yards per game & $32.21 \pm 21.48$ & $527.17 \pm 374.50$ \\
Rec yards per season & $425.14 \pm 320.67$ & $16.62 \pm 22.58$ \\
Touchdowns & $13.14 \pm 19.33$ & $6.84 \pm 2.92$ \\
Yards per target & $7.31 \pm 3.20$ & .62 \\
Fantasy points receiving yards & $205.59 \pm 304.93$ & .41 \\
Fantasy points touchdowns & $78.83 \pm 116.00$ & .68 \\
Fantasy points receptions & $150.24 \pm 217.10$ & .65 \\
Fantasy points total points & $434.66 \pm 634.65$ & $.59 .72 \pm 135.47$ \\
Points per game & $6.87 \pm 4.65$ & $180.21 \pm 243.42$ \\
Points per season & $90.75 \pm 69.50$ & $523.81 \pm 709.02$
\end{tabular}

ACL, anterior cruciate ligament; BMI, body mass index; NFL, National Football League; WR, wide receiver.

a major injury for any individual, but it can be potentially devastating and career-altering for many high-level NFL athletes. ${ }^{7}$ Between $21 \%$ and $37 \%$ of NFL athletes who suffer an ACL injury never appear in another NFL game. ${ }^{2,16}$ Those who do RTP have been shown to perform at a lower level on average than they did before injury. ${ }^{2}$ The physical demands imposed on NFL athletes predispose them to greater injury risk in comparison to the general population. A previous analysis of relative injury risk for each position demonstrated that certain skill position players, specifically WRs, tight ends, linebackers, RBs, and fullbacks, face a significantly greater ACL injury risk than the rest of the NFL between the 2010 and 2013 NFL seasons. ${ }^{9}$ However, despite the increased risk and consequences related to ACL injuries for these elite

Table 10. WR Pre-ACL Reconstruction Versus Post-ACL Reconstruction in Cases

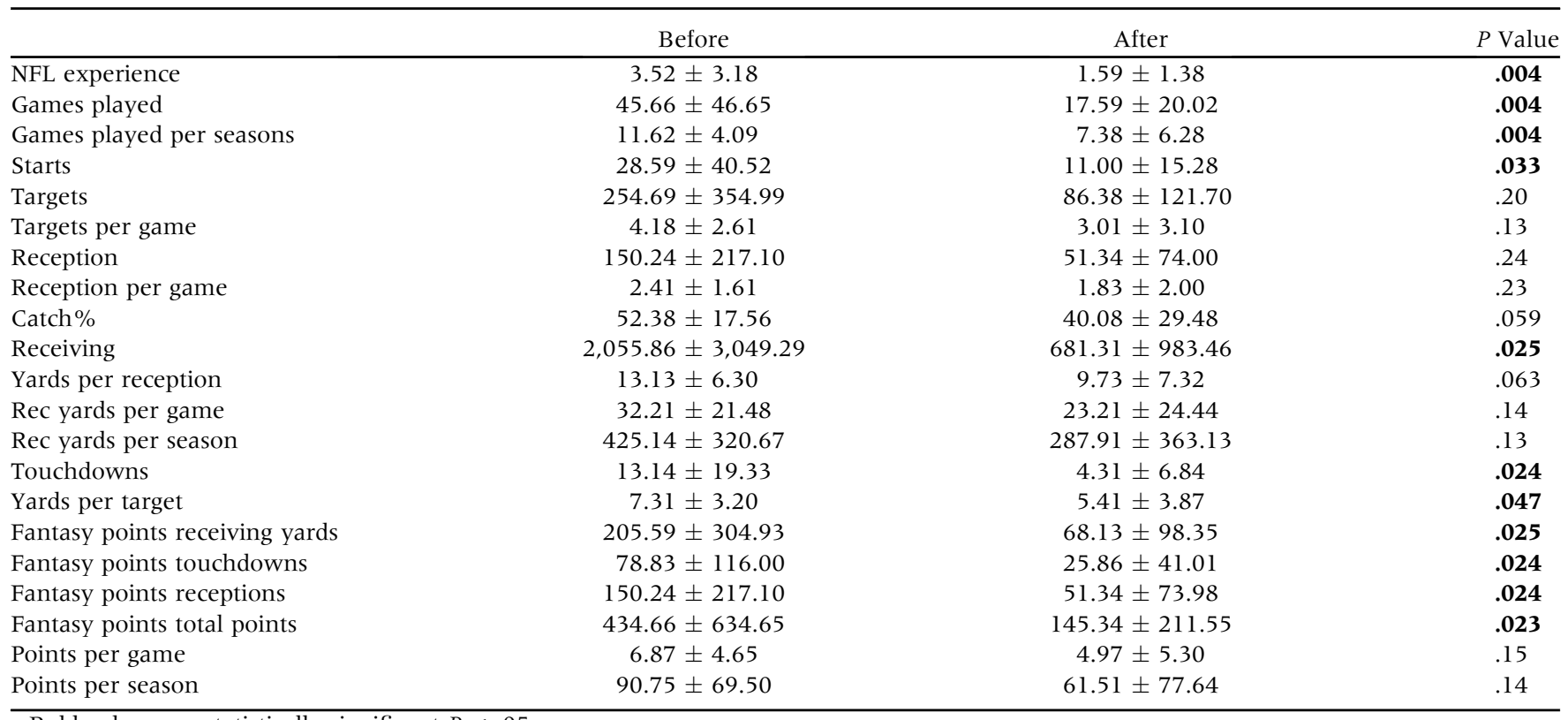

Bold values are statistically significant $P<.05$.

ACL, anterior cruciate ligament; NFL, National Football League; WR, wide receiver. 
Table 11. WR Post-ACL reconstruction in Cases Versus Postindex Year in Controls

\begin{tabular}{|c|c|c|c|}
\hline & After ACL & After Index Year & $P$ Value \\
\hline$\overline{\text { NFL experience }}$ & $1.59 \pm 1.38$ & $3.76 \pm 2.38$ & $<.0001$ \\
\hline Games played & $17.59 \pm 20.02$ & $50.21 \pm 37.34$ & $<.0001$ \\
\hline Games played per seasons & $7.38 \pm 6.28$ & $11.52 \pm 4.82$ & .007 \\
\hline Targets per game & $3.01 \pm 3.10$ & $4.64 \pm 2.67$ & .036 \\
\hline Reception & $51.34 \pm 74.00$ & $165.59 \pm 172.36$ & .002 \\
\hline Reception per game & $1.83 \pm 2.00$ & $2.71 \pm 1.61$ & .070 \\
\hline Yards per reception & $9.73 \pm 7.32$ & $11.86 \pm 4.47$ & .19 \\
\hline Rec yards per game & $23.21 \pm 24.44$ & $35.92 \pm 21.98$ & .042 \\
\hline Rec yards per season & $287.91 \pm 363.13$ & $471.43 \pm 321.25$ & .046 \\
\hline Touchdowns & $4.31 \pm 6.84$ & $13.55 \pm 14.41$ & .003 \\
\hline Yards per target & $5.41 \pm 3.87$ & $6.86 \pm 2.56$ & .098 \\
\hline Fantasy points receiving yards & $68.13 \pm 98.35$ & $213.98 \pm 214.40$ & .002 \\
\hline Points per season & $61.51 \pm 77.64$ & $101.04 \pm 68.49$ & .044 \\
\hline
\end{tabular}

Bold values are statistically significant $P<.05$.

ACL, anterior cruciate ligament; NFL, National Football League; WR, wide receiver.

athletes, the utility of fantasy football points as a simple outcome measure to compare performance in NFL offensive skill position players pre- and postACLR remains unknown.

Although it is not yet widely accepted in the medical community, we believe that fantasy football data are a true objective way to measure performance in highlevel professional athletes following an injury. It allows comparison of quantitative measures to determine whether an athlete can truly return to the same level of on-field performance following a major injury, such as an ACL tear. Additionally, fantasy football points are a recognized metric by athletes, as well as the general population. Therefore, the advantage of this study is that it allows the average American to easily discern the impact of ACLR versus traditional patient-reported

Table 12. WR Cases Versus Controls: Year One to Year Three

\begin{tabular}{lcrc}
\hline & Case & Control & $P$ Value \\
\hline 1st reception & $34.55 \pm 34.70$ & $39.59 \pm 25.58$ & .56 \\
1st receiving & $47.29 \pm 47.20$ & $57.13 \pm 38.49$ & .45 \\
1st TD fantasy points & $18.30 \pm 23.15$ & $21.52 \pm 19.61$ & .61 \\
1st total fantasy points & $100.14 \pm 102.65$ & $118.23 \pm 78.30$ & .51 \\
2nd reception & $32.21 \pm 32.87$ & $38.37 \pm 27.13$ & .55 \\
2nd receiving & $40.69 \pm 41.52$ & $49.69 \pm 36.72$ & .50 \\
2nd TD fantasy points & $16.71 \pm 16.50$ & $18.44 \pm 22.75$ & .80 \\
2nd Total FP & $89.62 \pm 88.55$ & $106.51 \pm 82.85$ & .56 \\
3rd reception & $35.14 \pm 20.16$ & $38.95 \pm 24.15$ & .76 \\
3rd receiving & $47.30 \pm 26.46$ & $48.48 \pm 30.62$ & .92 \\
3rd TD fantasy points & $13.71 \pm 13.29$ & $21.79 \pm 16.64$ & .22 \\
3rd Total FP & $96.16 \pm 57.43$ & $108.33 \pm 67.94$ & .66 \\
\hline
\end{tabular}

FP, fantasy points; TD, touchdowns; WR. Wide receiver. outcome measures. In addition, traditional patientreported outcome measures may be limited in their generalizability due to a ceiling effect in high-level professional athletes. Further research is warranted to better understand whether fantasy football points are a reliable metric of postinjury performance and correlates with traditional patient-reported outcome measures.

A recent study by Provencher et al. ${ }^{17}$ investigated the presence of symptomatic focal knee chondral injuries in NFL combine players using fantasy football points as one of their outcome measures. Although their study did not evaluate ACLR, the results were similar to our findings, as RBs and WRs with previously untreated knee chondral injuries were the only offensive players with significantly lower fantasy scores when compared with position-matched controls. ${ }^{17}$ QBs achieved statistically similar fantasy scores as the control group. Carey et $\mathrm{al}^{2}$ found that player performance in NFL RBs and WRs who sustained an ACL tear was reduced by one-third and that more than 1 in 5 players never returned to an NFL game after this injury. Contrarily, a study published in 2021 by Manoharan et al. ${ }^{18}$ found that although $61.8 \%$ of players $(64.5 \%$ of RBs, $60 \%$ of WRs) returned to play at a mean of 13.6 months following ACLR, RBs had an insignificant drop in production. However, WRs who successfully returned to play demonstrated significantly decreased receiving yards, receptions per game, and receiving touchdowns per season when compared with the control group. This was validated by our findings in a prior study, which showed that WRs had a significant decrease in targets, receptions, receiving 
Table 13. Fantasy Football Points Post-ACL Reconstruction Versus Postindex Year

\begin{tabular}{|c|c|c|c|c|c|c|}
\hline \multicolumn{4}{|c|}{ Per Game } & \multicolumn{3}{|c|}{ Career } \\
\hline Quarterbacks & $7.7 \pm 6.4$ & $10.1 \pm 4.0$ & .34 & $674.8 \pm 949.2$ & $1,162.9 \pm 1,116.1$ & .24 \\
\hline Wide Receivers & $5.0 \pm 5.3$ & $7.7 \pm 4.7$ & .042 & $145.3 \pm 211.5$ & $460.9 \pm 466.8$ & .002 \\
\hline
\end{tabular}

Bold values are statistically significant $P<.05$.

Values are expressed as means $\pm \mathrm{SD}$.

yards, and touchdowns along with playing 1.9 seasons fewer and fewer than half the number of games after undergoing ACLR compared with their postindex year-matched controls. ${ }^{8}$

While this study does not evaluate the percentage of players who return to competition following an ACL injury, our findings align with the aforementioned studies with regard to the decreased production of RBs and WRs following ACLR compared with their preinjury on-field production as well as postindex year players who did not suffer such an injury. Therefore, evaluating athletes' performance by the position they play is important because of the different demands placed on athletes based on the various on-field positions. Possible factors that may have allowed return to professional play, but at a less productive level, can likely be attributed to persisting knee pain, stiffness, loss of strength, deconditioning, reduced proprioception, and fear of reinjury.

With regard to QB play, Erickson et al. ${ }^{11}$ conducted a study that analyzed the performance and return-tosport after ACLR and found that there was also no significant in-game performance difference between preinjury play and control QBs (index year) following ACLR. Other studies have also found no performance differences between QBs who have native or reconstructed ACLs., ${ }^{3,19}$ Our results largely coincide with this finding; however, QBs in our study who underwent ACLR recorded fewer interceptions and rushing touchdowns during their lst season upon return-to-sport but were statistically similar 2nd and 3rd year in comparison to the index year controls. A possible reason for these findings in QBs may be due to the difference in physical demands of the position compared to the RB and WR position groups. RBs and WRs are more likely to jump, pivot, and cut over the course of live game play, which may be why these position groups saw a decline in production following ACLR. Multiple factors affect the ability to perform in an elite capacity after ACLR, including previous physical conditioning and access to optimal rehabilitation after undergoing surgery. ${ }^{19}$

Contrary to the present study, Keller et al. ${ }^{20}$ suggest that high-caliber athletes who participated in the NFL scouting combine between 2010 and 2014 can achieve equivalent levels of performance with no statistically significant differences in 40-yard dash time, vertical leap, broad jump, shuttle drill, and 3cone drill compared with matched controls. While these metrics may be similarly achieved in NFL skill position players post-ACLR compared with their pre-injury state; however, they do not necessarily translate to similar on-field production as our findings suggest.

\section{Limitations}

This study is not without limitations. This includes a retrospective study design, the relatively small sample size, and the use of the fantasy football points as a nonvalidated performance metric. Furthermore, it is difficult to truly identify a control group while equalizing "talent" and "skill," which partially determine productivity and objective outcomes of these NFL athletes. Since our data were based solely on public information available on the internet, we could not account for the differing techniques for ACLR. However, multiple previous studies have cited that the vast majority of NFL players who sustain an ACL tear undergo arthroscopically assisted single-bundle bonepatellar tendon-bone reconstruction. ${ }^{1,21,22}$ Thus, regardless of the possible variables in graft choice, surgical technique, and surgeon experience, it is likely that the majority of the athletes whom we reviewed had similar ACLR techniques and graft choices. Validated outcome scores evaluating high-level sports performance are still lacking; therefore, the performance measure used in this study may not truly reflect the actual on-field impact of the player. However, an advantage of using fantasy football points as an outcome tool is the consistent follow-up since the statistical data are documented annually and widely available to the public. Another limitation of our methodology is that injured players have already played a portion of their careers, and thus, the fewer number of games played after surgery may be due to the natural process of players nearing the end of their careers and not due to inferiority of their reconstructed knees. Despite these limitations, the present study suggests that RBs and WRs have a significantly decreased performance output upon returning from ACLR in comparison to their preinjury status, as well as postindex year counterparts, who did not suffer an ACL injury. 


\section{Conclusion}

QBs did not have a statistically significant decrease in performance following ACL reconstruction based on fantasy football performance. Conversely, both running backs and wide receivers had decreased per game and career performance post-ACL reconstruction based on their fantasy football statistics.

\section{References}

1. Schrock JB, Carver TJ, Kraeutler MJ, McCarty EC. Evolving treatment patterns of NFL players by orthopaedic team physicians over the past decade, 2008-2016. Sport Heal A Multidiscip Approach 2018;10:453-461. doi:10. $1177 / 1941738118758312$.

2. Carey JL, Huffman GR, Parekh SG, Sennett BJ. Outcomes of anterior cruciate ligament injuries to running backs and wide receivers in the National Football League. Am J Sports Med 2006;34:1911-1917. doi:10.1177/0363546506290186.

3. Brophy RH, Lyman S, Chehab EL, Barnes RP, Rodeo SA, Warren RF. Predictive value of prior injury on career in professional American football is affected by player position. Am J Sports Med 2009;37:768-775. doi:10.1177/ 0363546508329542.

4. Brophy RH, Gill CS, Lyman S, Barnes RP, Rodeo SA, Warren RF. Effect of anterior cruciate ligament reconstruction and meniscectomy on length of career in National Football League athletes. Am J Sports Med 2009;37: 2102-2107. doi:10.1177/0363546509349035.

5. Kluczynski MA, Kelly WH, Lashomb WM, Bisson LJ. A systematic review of the orthopaedic literature involving National Football League players. Orthop J Sport Med 2019;7: 232596711986435. doi:10.1177/2325967119864356.

6. Rothenberg P, Grau L, Kaplan L, Baraga MG. Knee injuries in American football: An epidemiological review. Am J Orthop (Belle Mead NJ) 45:368-373. http://www.ncbi. nlm.nih.gov/pubmed/27737282.

7. Mai HT, Alvarez AP, Freshman RD, et al. The NFL orthopaedic surgery outcomes database (NO-SOD). Am J Sports Med 2016;44:2255-2262. doi:10.1177/0363546 516651426.

8. Burgess CJ, Stapleton E, Choy K, Iturriaga C, Cohn RM. Decreased performance and return to play following anterior cruciate ligament reconstruction in National Football League wide receivers. Arthrosc Sport Med Rehabil 2021;3:e455-e461.

9. Dodson CC, Secrist ES, Bhat SB, Woods DP, Deluca PF. Anterior cruciate ligament injuries in National Football League athletes from 2010 to 2013. Orthop J Sport Med 2016;4: 232596711663194. doi:10.1177/2325967116631949.

10. Cinque ME, Hannon CP, Bohl DD, et al. Return to sport and performance after anterior cruciate ligament reconstruction in National Football League linemen. Orthop $J$ Sport Med 2017;5:232596711771168. doi:10.1177/23259 67117711681.
11. Erickson BJ, Harris JD, Heninger JR, et al. Performance and return-to-sport after ACL reconstruction in NFL quarterbacks. Orthopedics 2014;37:e728-e734. doi:10. 3928/01477447-20140728-59.

12. Read CR, Aune KT, Cain EL, Fleisig GS. Return to play and decreased performance after anterior cruciate ligament reconstruction in National Football League defensive players. Am J Sports Med 2017;45:1815-1821. doi:10. $1177 / 0363546517703361$.

13. FSGA. Fantasy Sports \& Gaming Association - Industry Demographics, https://thefsga.org/industry-demo graphics/. Accessed October 4, 2021.

14. ESPN. Fantasy Football Scoring. Published 2021, https:// support.espn.com/hc/en-us/articles/360003914032-ScoringFormats. Accessed October 4, 2021.

15. Signore M. Fantasy Football for Dummies. New York: John Wiley \& Sons, 2007, https://books.google.com/books?

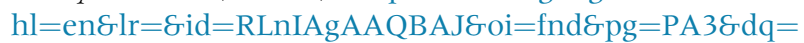
fantasy + football + point + \&ots $=$ p3zSkOnHjF\&sig $=7 \mathrm{nEnBC}$ _h0oQjKaxWcyjFcKExbOI\#v=onepage\&q=fantasy football point\&f=false.

16. Meredith SJ, Rauer T, Chmielewski TL, et al. Return to sport after anterior cruciate ligament injury: Panther Symposium ACL Injury Return to Sport Consensus Group. Orthop J Sport Med 2020;8:232596712093082. doi: $10.1177 / 2325967120930829$.

17. Provencher MT, Chahla J, Cinque ME, et al. Symptomatic focal knee chondral injuries in National Football League combine players are associated with poorer performance and less volume of play. Arthrosc J Arthrosc Relat Surg 2018;34:671-677. doi:10.1016/j.arthro.2017.08.300.

18. Manoharan A, Barton D, Khwaja A, Latt LD. Return to play rates in NFL wide receivers and running backs after ACL reconstruction: An updated analysis. Orthop J Sport Med 2021;9:232596712097474. doi:10.1177/232596 7120974743.

19. Shah VM, Andrews JR, Fleisig GS, McMichael CS, Lemak LJ. Return to play after anterior cruciate ligament reconstruction in National Football League athletes. Am J Sports Med 2010;38:2233-2239. doi:10.1177/ 0363546510372798.

20. Keller RA, Mehran N, Austin W, Marshall NE, Bastin K, Moutzouros V. Athletic performance at the NFL scouting combine after anterior cruciate ligament reconstruction. Am J Sports Med 2015;43:3022-3026. doi:10.1177/ 0363546515608482.

21. Erickson BJ, Harris JD, Fillingham YA, et al. Anterior cruciate ligament reconstruction practice patterns by NFL and NCAA Football Team physicians. Arthrosc J Arthrosc Relat Surg 2014;30:731-738. doi:10.1016/j.arthro.2014. 02.034 .

22. Bradley JP, Klimkiewicz JJ, Rytel MJ, Powell JW. Anterior cruciate ligament injuries in the National Football League. Arthrosc J Arthrosc Relat Surg 2002;18:502-509. doi:10. 1053/jars.2002.30649. 